Supporting Information

\title{
Mechanistic study of membrane disruption by antimicrobial methacrylate random copolymers by the single giant vesicle method
}

Manami Tsukamoto, ${ }^{a}$ Emanuele Zappala ${ }^{b \dagger}$ Gregory A. Caputo, ${ }^{c}$ Jun-ichi Kikuchi, ${ }^{a}$ Kayvan Najarian, ${ }^{b}$ Kenichi Kuroda, ${ }^{*, d}$ Kazuma Yasuhara* ${ }^{*}$, e

a) Division of Materials Science, Graduate School of Science and Technology, Nara Institute of Science and Technology, 8916-5 Takayama, Ikoma, Nara 6300192, Japan.

E-mail: yasuhara@ms.naist.jp; Fax:+81-743-72-6099; Tel: +81-743-72-6091

b) Department of Computational Medicine and Bioinformatics, University of Michigan, Ann Arbor, MI 48109-2800, U.S.A.

c) Department of Chemistry and Biochemistry, Rowan University, Glassboro, NJ 08028, U.S.A.

d) Department of Biologic and Materials Sciences, University of Michigan School of Dentistry, 1011 N. University Ave., Ann Arbor, MI 48109, U.S.A.

E-mail: kkuroda@umich.edu; Fax: +1-734-647-2110; Tel: +1-734-936-1440

e) Center for Digital Green-innovation, Nara Institute of Science and Technology, 8916-5

Takayama, Ikoma, Nara 6300192, Japan.

$\dagger$ Present address: Institute of Mathematics and Statistics, University of Tartu, Tartu, 51009, Estonia.

\section{Synthesis and characterization of cationic methacrylate random copolymer}

$\mathrm{N}$-(tert-butoxycarbonyl)aminoethylmethacrylate (Boc-AEMA) and the cationic polymethacrylate derivatives were prepared as previous described. ${ }^{1}$ The polymerization was carried out in acetonitrile using 2,2'-azodiisobutyronitrile (AIBN) and methyl 3-mercaptopropionate (MMP) as an initiator and a chain transfer agent, respectively. As a representative example, the $\mathrm{PM}_{57}$ polymer was prepared by the following procedure. Boc-AEMA $(19.4 \mathrm{mg}, 0.09 \mathrm{mmol})$ and methyl methacylate $(11.8 \mu \mathrm{L}, 0.21$ $\mathrm{mmol})$ was dissolved in anhydrous acetonitrile $(300 \mu \mathrm{L})$ in a glass test tube. Then, MMP $(54.1 \mathrm{mg} / \mathrm{mL}$, $100 \mu \mathrm{L}, 0.045 \mathrm{mmol})$ and $\operatorname{AIBN}(4.93 \mathrm{mg} / \mathrm{mL}, 100 \mu \mathrm{L}, 0.003 \mathrm{mmol})$ were added into test tube sealed with a rubber septum and bubbled with $\mathrm{N}_{2}$ gas for 5 minutes. The polymerization was carried out by stirring the reaction mixture at $65^{\circ} \mathrm{C}$ overnight. After the polymerization, the solvent was evaporated under reduced pressure. The crude mixture of the polymer was dissolved in trifluoroacetic acid (500 $\mu \mathrm{L}$ ) and allowed to stir at room temperature for 1 hour to deprotect Boc group. Obtained oily residue was dissolved in a small amount of methanol and added to ice-cold diethyl ether. The resultant precipitate was collected by centrifugation and lyophilized from aqueous solutions to give cationic copolymers as a white powder. ${ }^{1} \mathrm{H}$ NMR analysis provided determination of the degree of polymerization (DP) and the mole percentage of alkyl methacrylate, $f_{\mathrm{HB}}$, of polymers. The integration of the signals from the following groups was used for the characterization of polymers : Methyl group for $\mathrm{PM}_{34}$ and $\mathrm{PM}_{57}(3.6 \mathrm{ppm}, 3 \mathrm{H})$ or methylene group for $\mathrm{PB}_{36}(4.0 \mathrm{ppm}, 2 \mathrm{H})$ in hydrophobic sidechains, the methylene in cationic sidechains $(4.2 \mathrm{ppm} 2 \mathrm{H})$ and backbone methylenes in the terminal group (2.9-2.5 ppm, 7H). Representative ${ }^{1} \mathrm{H}$ NMR (400 MHz, Methanol-d4, TMS) for $\mathrm{PM}_{57}$ 
$\left(f_{\mathrm{HB}}=0.57\right.$, and $\left.\mathrm{DP}=15\right): \delta 4.2(\mathrm{~m}, 12.9 \mathrm{H}), 3.6(\mathrm{~m}, 28.7 \mathrm{H}), 3.3(\mathrm{~m}, 12.9 \mathrm{H}), 2.9-2.5(\mathrm{~m}, 7 \mathrm{H}), 2.3-1.8$ (m, 30H), 1.5-0.7 (m, 45H).

\section{Calculation of total cross-sectional area of pores formed by the polymer}

We have calculated the total cross-sectional area of the pores formed by the $\mathrm{PB}_{36}$ at the concentration of $66.7 \mu \mathrm{g} \cdot \mathrm{mL}^{-1}$ based on the result of sucrose leakage as follows. Since we assume that the entrapped molecule is released by diffusion-mediated process, the leakage kinetics follows the Fick's first law. The rate of marker release is expressed by employing the diffusion coefficient of entrapped marker, $D$, the volume of vesicles, $V$, membrane thickness, $\delta$, concentration of the marker inside the vesicle, $C$, and the total area of pore, $P$ as follows. ${ }^{3}$

$-\mathrm{d} C / \mathrm{d} t=D p /(V \delta) \cdot C \quad-\quad(\mathrm{S} 1)$

Since the concentration of the marker inside the vesicle is sufficiently higher than that of outside the vesicle, we can regard the concentration of the marker at the outside the vesicle to be zero. Thus, the concentration of the marker remaining inside the vesicle at time $t$ is given by

$C=C_{0} \cdot \exp (-D p /(V \delta) \cdot t) \quad-$

where $C_{0}$ corresponds to the initial concentration of the marker entrapped in a vesicle.

The total cross-sectional area of the pore formed on single GUV was estimated by introducing a time required to release the $50 \%$ of entrapped marker, $t_{50}$, where $C=1 / 2 C_{0}$.

$\exp \left(-D p /(V \delta) \cdot t_{50}\right)=1 / 2$

$p=\ln 2 \cdot(V \delta) /\left(D t_{50}\right) \quad-$

The total area of the pore, $p$ was calculated to be $1.6 \times 10^{2} \mathrm{~nm}^{2}$ by substituting each of the following parameters into the equation S3. The diffusion coefficient of the sucrose $D_{\text {sucrose }}=5.2 \times 10^{-10}\left(\mathrm{~m}^{2} / \mathrm{sec}\right)$ ${ }^{4}$ and membrane thickness $\delta=4.165 \times 10^{-9}(\mathrm{~m})^{5}$ were referred to the literatures. The volume of the vesicle $V=4 / 3 \pi \times\left(7.5 \times 10^{-6}\right)^{3}\left(\mathrm{~m}^{3}\right)$ and $t_{50}=69(\mathrm{sec})$ were obtained from the image showin in Fig. 4D.

\section{Simulation of RITC-dextran leakage}

We have simulated the time-course of the RITC-dextran release by introducing the diffusion coefficient of $70 \mathrm{kDa}$ dextran $D_{\text {dextran }}=37 \times 10^{-12}\left(\mathrm{~m}^{2} / \mathrm{sec}\right) .{ }^{6}$ The fraction of retained dextran, $F$ can be calculated as follows. 
$F=\exp \left(-D_{\operatorname{dextran}} \cdot p /(V \delta) \cdot t\right) \quad-\quad(\mathrm{S} 4)$

Here, we used the parameters $p, V$, and $\delta$ shown above. Obtained time-course according to this calculation is shown in the Fig. S4. Experimental data on the fraction of entrapped dextran was also plotted in Fig. S5 that was obtained by $\left(\mathrm{I}_{\mathrm{out}}-\mathrm{I}_{\mathrm{in}, \mathrm{t}}\right) /\left(\mathrm{I}_{\mathrm{out}}-\mathrm{I}_{\mathrm{in}, 0}\right)$ where $\mathrm{I}_{\mathrm{out}}, \mathrm{I}_{\mathrm{in}, 0}$, and $\mathrm{I}_{\mathrm{in}, \mathrm{t}}$ are the fluorescence intensities corresponding to outside of the GUV, the inside of the GUV before the addition of polymer, and time $t$ after the addition of polymers, respectively. 


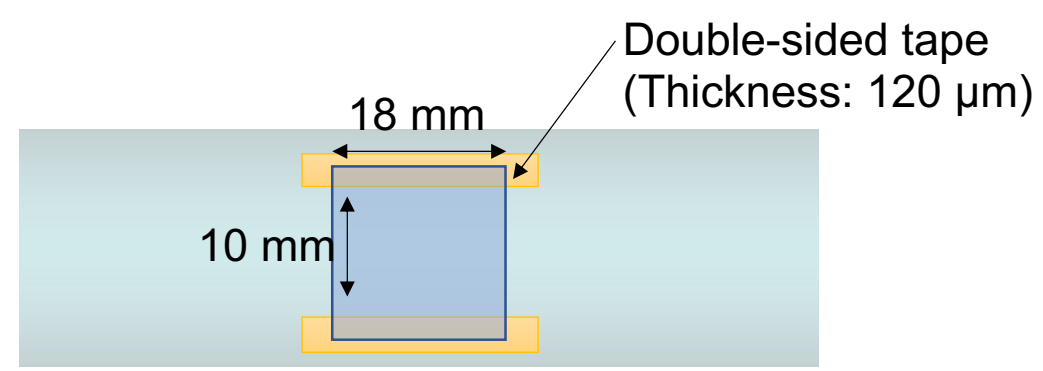

Figure S1. Schematic image of the chamber for the GUV observation.

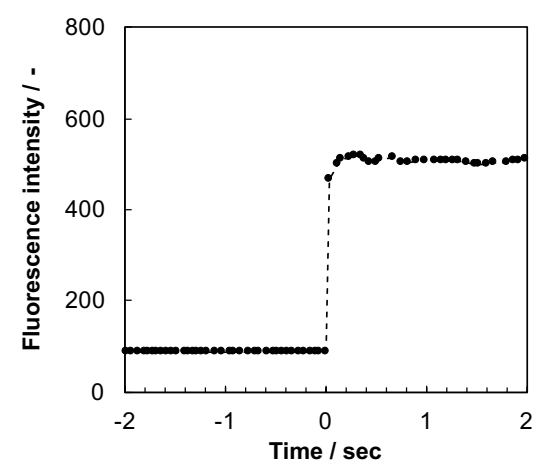

Figure S2. Time-course of the FITC-dextran fluorescence measured at $100 \mu \mathrm{m}$ away from the injection pipette. FITC-dextran was injected at 0 second. The fluorescence intensity was obtained by the image analysis using and ImageJ software.

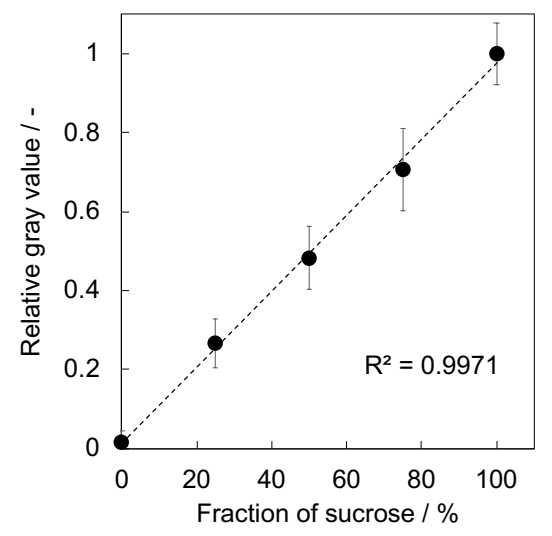

Figure S3. Calibration curve for the relative gray value vs fraction of sucrose entrapped in GUVs. 


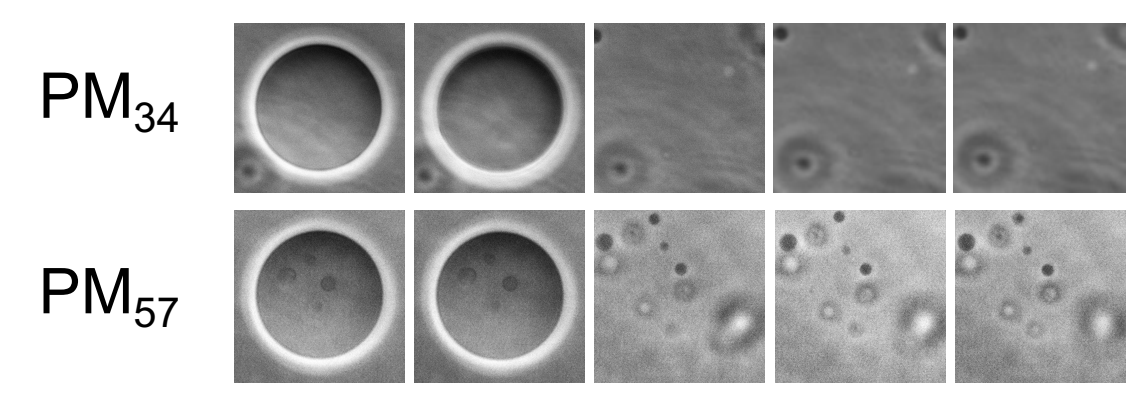

Figure S4. Time lapse image of the burst of POPE/POPG GUVs by the polymer at the concentration of $66.7 \mu \mathrm{g} \cdot \mathrm{mL}^{-1}$. The time interval of each image is 0.2 second.

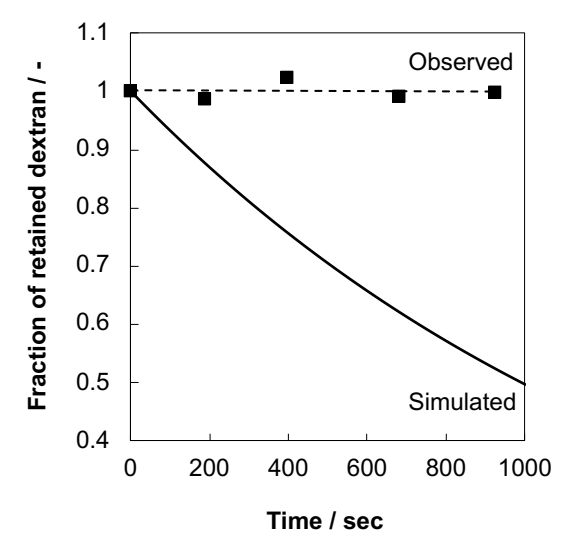

Figure S5. Time course of the RITC-dextran concentration inside the POPE/POPG GUV after the treatment of $\mathrm{PB}_{33}$ polymer at the concentration of $66.7 \mu \mathrm{g} \cdot \mathrm{mL}^{-1}$. Simulation curve was obtained by the equation $\mathrm{S} 4$.

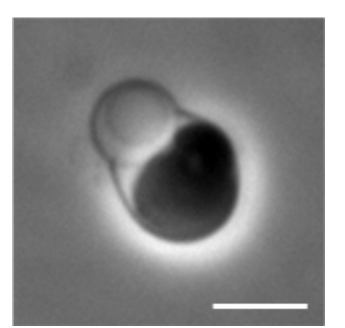

Figure S6. Phase contrast microscopic image for the deformation of POPE/POPG GUV entrapping RITC-dextran induced by $\mathrm{PB}_{33}$ polymer at the concentration of $66.7 \mu \mathrm{g} \cdot \mathrm{mL}^{-1}$ 


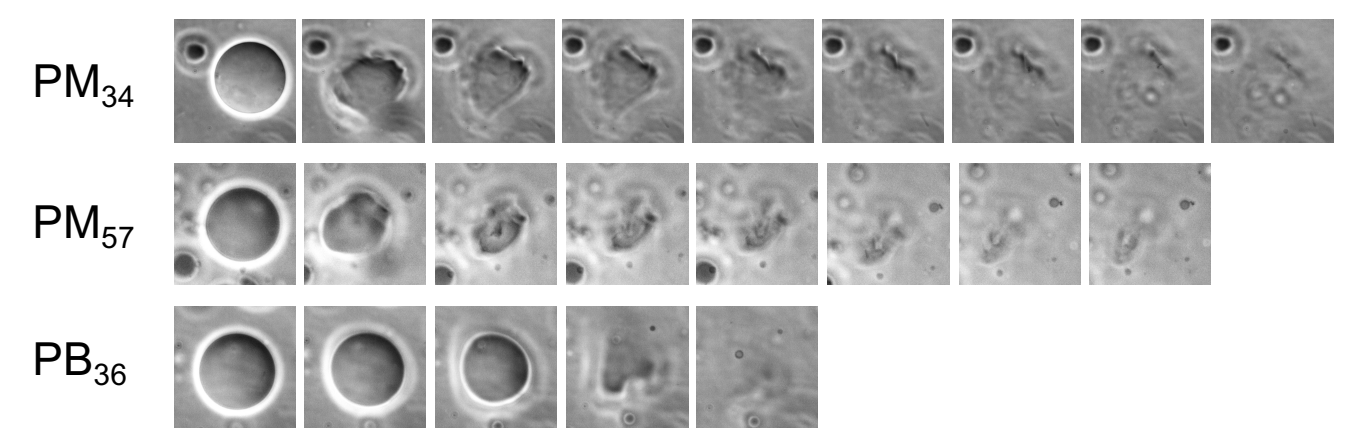

Figure S7. Time lapse image of the deformation of POPE/POPG GUVs by the polymer at the concentration of $166.8 \mu \mathrm{g} \cdot \mathrm{mL}^{-1}$. The time interval of each image is 0.2 second.
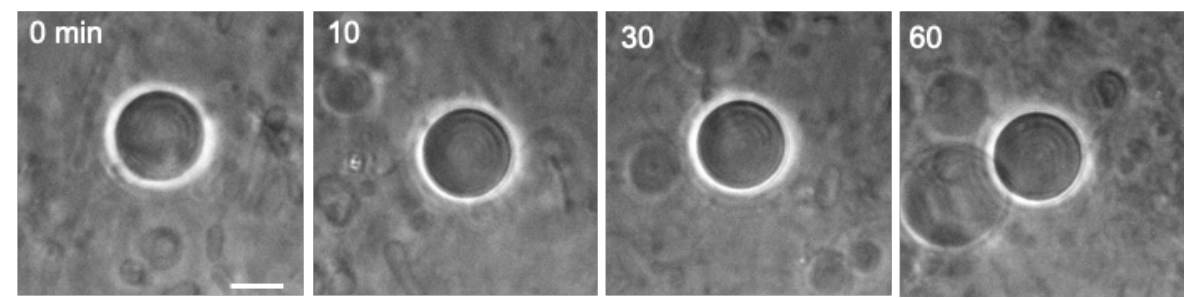

Figure S8. Long time observation of POPC GUV after the addition of $\mathrm{PM}_{34}$ at the concentration of $66.7 \mu \mathrm{g} \cdot \mathrm{mL}^{-1}$. Bar $=10 \mu \mathrm{m}$

\section{References:}

1. Kuroda, K.; DeGrado, W., Amphiphilic polymethacrylate derivatives as antimicrobial agents. J. Am. Chem. Soc. 2005, 127 (12), 4128-4129.

2. Kuroda, K.; Caputo, G.; DeGrado, W., The Role of Hydrophobicity in the Antimicrobial and Hemolytic Activities of Polymethacrylate Derivatives. Chem. Eur. J. 2009, 15 (5), 1123-1133.

3. Jacobs, M. H., Diffusion processes. Springer, 2013.

4. Lide, D. R., CRC handbook of chemistry and physics, 1999-2000. CRC Press: Boca Raton, 1999.

5. Mukherjee, S.; Kar, R.; Nanga, R.; Mroue, K.; Ramamoorthy, A.; Bhunia, A., Accelerated molecular dynamics simulation analysis of MSI-594 in a lipid bilayer. Phys. Chem. Chem. Phys. 2017, 19 (29), 19289-19299.

6. Hristova, K.; Selsted, M.; White, S., Critical role of lipid composition in membrane permeabilization by rabbit neutrophil defensins. J. Biol. Chem. 1997, 272 (39), 24224-24233. 